\title{
Occurrence of Ureaplasma urealyticumin in Women in the Northeast of Iran: Characterization of Resistance Trends
}

\author{
Jalal Mardaneh ${ }^{1}$, Alireza Mohammadzadeh', Mahdieh Sadat Alavi ${ }^{2}$, Mahdieh Zendehdel ${ }^{2}$, Narjes \\ Bahri $^{3}$, Mehrnaz Mehraban ${ }^{4,5}$, Abdollah Ardebili ${ }^{6}$, Gholamreza Pouladfar ${ }^{7}$, Mojtaba Anvarinejad ${ }^{6 *}$
}

\begin{abstract}
Objectives: The present study surveyed the prevalence of antibiotic resistance among Ureaplasma urealyticum in isolates from Gonabad (in the northeast of Iran) including susceptibility testing for U. urealyticum to different antibiotics.

Materials and Methods: In this research, a total of 95 vaginal swab specimens were aseptically collected from women who were admitted to the Bohlool Teaching Hospital and Jahad Daneshgahi Center from April 2016 to April 2017. Culture and subsequently antibiotic susceptibility testing were performed according to the Mycoplasma IST 2 kit. Then the cupules were read and interpreted in 24 and 48 hours according to kit guidelines.

Results: In the studied patients, $38(40.4 \%), 12(12.8 \%)$, and $11(11.7 \%)$ cases were single positive for U. urealyticum, single positive for Mycoplasma hominis (M. hominis), and dually positive for U. urealyticum and M. hominis, respectively. The positive rates of genital U. urealyticum in the symptomatic and asymptomatic groups were $86.8 \%$ and $13.2 \%$, respectively. The highest positive rate $(42.1 \%)$ was found in the $26-30$-year-old group. In addition, tetracycline (TET) and doxycycline (DOT) were the most effective antibiotics against isolates, and one strain was multi-drug resistant. The $U$. urealyticum resistance rates were more than $39 \%$ to erythromycin and pristinamycin, and more than $55 \%$ to ciprofloxacin. All U. urealyticum isolates with $<104 \mathrm{CFU} /$ specimen were sensitive to all tested drugs.

Conclusions: Although the emerging resistance to TETs among our isolates is alarming, these data show that the standard therapeutic regimen for urogenital infections caused by $U$. urealyticum is DOT, TET, and clarithromycin, leading to better outcomes in most respective patients.

Keywords: Women, Urogenital infection, Ureaplasma urealyticum, Antibiotic susceptibility pattern
\end{abstract}

\section{Introduction}

Ureaplasma urealyticum is the smallest and simplest selfreplicating bacterium belonging to the class Mollicutes and is only bounded by the bacterial membrane (1). This organism is highly fastidious and completely dependent on host biosynthetic precursors (2). It is frequently isolated from human amniotic fluid and the placenta. Approximately $40-80 \%$ of healthy adult women may be the carrier of Ureaplasmas in their cervix and vagina. The occurrence of Ureaplasma in the healthy men's lower urogenital tract is somewhat less (3).

Ureaplasma urealyticum is easily transmitted vertically and venereally either at the delivery of the neonate or in utero (2). It is in the neonatal respiratory tract or a colonizer of the female and male urogenital systems. The pathogenicity of this bacterium in urethritis has been documented in some studies (4,5). Ureaplasma species may cause or be related to a variety of clinical manifestations in adults, including meningitis, preterm birth, postpartum endometritis, urethritis, chorioamnionitis, chronic lung disease in neonates, abscesses, arthritis, bacteremia, and pneumonia $(2,6)$.

The systemic spread of this bacterium is possible in the immunosuppressed condition beyond the neonatal period, including hypogammaglobulinemia (2). $U$. urealyticum causes nongonococcal urethritis in humans and has also been associated with chorioamnionitis, abortion, infertility, low-weight infants, premature rupture of membranes, preterm labor, and preterm delivery and leads to apparently normal pregnancy outcomes (7).

After the diagnosis of Ureaplasma infection, selective drugs are confined for treatment. The absence of the 


\section{Key Message}

- The tetracycline and erythromycin resistant strains emerging in our investigation are really alarming. The important risk for drug resistance emerging in Ureaplasma uralyticum has potential clinical sequels in therapeutic guidelines.

cell wall renders $U$. urealyticum intrinsically resistant to all effective drugs on the bacterial cell wall such as glycopeptide and $\beta$-lactam antibiotics (8). According to García-Castillo et al study (9), antibiotic classes which are recognized to be effective against Ureaplasma include macrolides, quinolones, and tetracyclines (TETs). These therapy choices are further limited in the neonates for whom the only recognized treatment is to use macrolides because of the related toxicity of TETs and quinolones (10).

Considering the above-mentioned explanations, the main goal of the present research was to find the role of $U$. urealyticum in clinical problems occurring in the genitourinary tract of women by the culturing method and to determine the antibiotic susceptibility patterns of the isolates. The study further investigated the prevalence of resistance to antibiotics among $U$. urealyticum strains isolated from Gonabad (Khorasan Razavi province, in the northeast of Iran). Finally, in vitro susceptibility testing was performed for $U$. urealyticum to different antibiotics.

\section{Materials and Methods}

\section{Clinical Specimen Collection}

The presentstudy was carried out after approval of the Ethics Committee of Gonabad University of Medical Sciences (The code number IR.GMU.REC.1393. 12727.4.5). In total, 95 vaginal swab samples were aseptically obtained from women admitted to the Bohlool Teaching Hospital and Jahad Daneshgahi Center, in Gonabad from April 2016 to April 2017. The specimens were collected from married and unmarried women either pregnant or nonpregnant and included vaginal Dacron swabs containing two samples from each woman. The Mycoplasma R1 vial was allowed to reach laboratory temperature. Afterward, Dacron swabs were directly placed in the Mycoplasma R1 solution (a special transport medium) to maintain the swab wet. The inoculated vial of Mycoplasma R1, coated in an ice bag and protected from the light, was transported to the clinical microbiology laboratory for culture and subsequently antibiotic susceptibility testing. The transport medium vial was mixed, and subsequently,3 milliliters of the inoculated Mycoplasma R1 solution vial was transferred into the Mycoplasma R2 vial shaken on a vortex to ensure that the lyophilization pellet was quite dissolved. This inoculum was applied to inoculate the Mycoplasma IST 2 strip, and then it was allowed to reach laboratory temperature. The diagnostic strip was removed from its packaging. Immediately, $55 \mu \mathrm{L}$ of the broth medium was dispensed into each of the 22 test cupules on the Mycoplasma IST 2 strip by the pipette. Next, 2 drops of the mineral oil were added to each cupule and the lid was placed on the strip. The remaining strip and the broth in the Mycoplasma R2 vial were incubated at $36^{\circ} \mathrm{C} \pm 2$ for 24 and 48 hours. The change in the color of the UreaArginine LYO 2 broth was read after 24 and 48 hours of incubation. Finally, the cupules were read and interpreted in 24 and 48 hours except for Ureaplasma spp. $\geq 10^{4} \mathrm{CFU} /$ specimen which was read in 24 hours.

\section{Determination Antibiotic Susceptibility Patterns of} Ureaplasma urealyticum

The Mycoplasma IST 2 diagnostic kit was applied for the characterization of antibiotic susceptibility patterns. The broth medium prepares optimum replication and growth conditions for U. urealyticum. This strip (cupules) provides simultaneous results for the susceptibility testing of isolates with 9 different antibiotics. These antibiotics included doxycycline (DOT, concentrations of 4 and $8 \mathrm{mg} / \mathrm{L}$ ), josamycin (JOS, concentrations of 2 and 8 $\mathrm{mg} / \mathrm{L}$ ), ofloxacin (OFL, concentrations of 1 and $4 \mathrm{mg} / \mathrm{L}$ ), erythromycin (ERY, concentrations of 1 and $4 \mathrm{mg} / \mathrm{L}$ ), TET (concentrations of 2 and $8 \mathrm{mg} / \mathrm{L}$ ), ciprofloxacin (CIP, concentrations of 1 and $2 \mathrm{mg} / \mathrm{L}$ ), azithromycin (AZI, concentrations of 0.12 and $4 \mathrm{mg} / \mathrm{L}$ ), clarithromycin (CLA, concentrations of 1 and $4 \mathrm{mg} / \mathrm{L}$ ), and pristinamycin (PRI, $2 \mathrm{mg} / \mathrm{L})$.

Multi-drug Resistant $U$. urealyticum Isolate Detection Multi-drug-resistant $U$. urealyticum isolates were defined to be resistant to at least three antibiotics indifferent classes of antimicrobial drugs (including ERY, CIP, and TET) by the Kirby-Bauer disk diffusion technique. The results were analyzed in accordance with the Clinical and Laboratory Standards Institute (CLSI) (2015) guidelines.

\section{Statistical Analysis}

The results were analyzed by SPSS 16 statistical software. $P$ values $\leq 0.05$ were considered statistically significant. Standard deviations and means were calculated as required for numerical variables.

\section{Results}

In general, 95 vaginal swab specimens submitted to the clinical microbiology laboratory in the Mycoplasma R1 solution for $U$. urealyticum culture were evaluated in the present study. The titer was low in 11 (11.5\%) samples, thus the color change was observed in the Mycoplasma R1 transport medium vial only and not in the control cupule on the strip (the titer of the bacteria in the sample is too low to produce the color change). The analysis of culture results revealed that the prevalence rate of $U$. urealyticum infection was $40.4 \%$ (95\% CI 39.45\%-41.35\%). Based on the results, 11 out of $38 \mathrm{U}$. urealyticum infected women 
had a co-infection with Mycoplasma hominis. Table 1 presents the key characteristics of the study population regarding sexual behavior and sociodemographic status.

In these studied patients, $12(12.8 \%)$ and $38(40.4$ $\%$ ) cases were single positive for $M$. hominis and $U$. urealyticum, respectively, and 11 (11.7\%) cases were dually positive for M. hominis and U. urealyticum (Table 1). The bacteria concentration in $33(35.1 \%)$ isolates was higher than $10^{4}$ while it was lower than $10^{4}$ in $5(5.3 \%)$ isolates. There was no significant relation between U. urealyticum counts and menstrual cycles.

However, a significant relationship was found between $U$. urealyticum infected patients and their husbands' educational level $(P=0.04)$. Respectively, the positive rates of genital $U$. urealyticum in the asymptomatic and symptomatic groups were $13.2 \%$ and $86.8 \%$, respectively. In the univariate analysis of socio-demographical associated with $U$. urealyticum in the studied women, the increasing weight of patients was especially associated with a greater risk of being infected. The total positive rates of genital $U$. urealyticum in 26-35-year-old individuals were relatively higher compared with the other age groups and drastically reduced in womenover 46 years. The highest positive rate (42.1\%) was found in the 26-35 year age group. However, the positive rates in women living with their husbands and those living in separation revealed a significant difference $(P=0.05)$.

Tables 2 and 3 provide data on the antibiotic susceptibility patterns of $U$. urealyticum isolates. TET and DOT were the most effective drugs against those strains. For $U$. urealyticum, the obtained rates of susceptibility to some antibiotics by the IST 2 diagnostic kit included DOT (92.1\%), TET (92.1\%), azithromycin (AZT, 65.8\%), OFL (65.8\%), ERY (60.5\%), and PRI (60.5\%). In the analysis of the isolates, one strain was multi-drug-resistant. The resistance rates of $U$. urealyticum were more than 39\% to ERY and PRI, and more than 55\% to CIP while the rates were lower than $8 \%$ to DOT and TET (Table 2). Based on the results, $28.9 \%$ of the strains revealed decreased response (intermediate susceptible) to the more newly presented quinolones (OFL). None of the isolates was intermediate susceptible to DOT. Among the studied isolates, the higher intermediate response to drugs was shown for CIP $(36.8 \%, \mathrm{n}=14)$. Among the macrolide class of antibiotics, CLA was the most effective one against the isolates. All $U$. urealyticum isolates with $<10^{4} \mathrm{CFU} /$ specimen were susceptible to all tested antibiotics.

\section{Discussion}

The current study sought to determine the prevalence, antimicrobial susceptibility patterns, resistance profiles, and the multidrug resistance of $U$. urealyticum recovered from patient samples in Gonabad in the north-eastern of Iran.

In the past decade, $U$. urealyticum received further attention because of its association with preterm
Table 1. Demographic Analysis of Ureaplasma urealyticum Positive Patients $(\mathrm{n}=38)$

\begin{tabular}{|c|c|}
\hline Variable & No. $(\%)$ \\
\hline \multicolumn{2}{|l|}{ Age (y) } \\
\hline $15-25$ & $10(26.3)$ \\
\hline $26-35$ & $16(42.1)$ \\
\hline $36-45$ & $10(26.3)$ \\
\hline$\geq 46$ & $2(5.3)$ \\
\hline \multicolumn{2}{|l|}{ Weight (kg) } \\
\hline $31-40$ & $1(2.6)$ \\
\hline $41-50$ & $3(7.9)$ \\
\hline $51-60$ & $8(21.1)$ \\
\hline$\geq 61$ & $26(68.4)$ \\
\hline \multicolumn{2}{|l|}{ Job } \\
\hline Housewife & $31(81.6)$ \\
\hline Jobholder & $7(18.4)$ \\
\hline \multicolumn{2}{|l|}{ Education level } \\
\hline Cycles & $12(31.6)$ \\
\hline Diploma & $16(42.1)$ \\
\hline Collegiate & $10(26.3)$ \\
\hline \multicolumn{2}{|l|}{ Infertility } \\
\hline Yes & $0(0)$ \\
\hline No & $38(100)$ \\
\hline \multicolumn{2}{|l|}{ Urinary tract infection } \\
\hline Yes & $12(31.6)$ \\
\hline No & $26(68.4)$ \\
\hline \multicolumn{2}{|l|}{ Genital infection } \\
\hline Yes & $15(39.5)$ \\
\hline No & $23(60.5)$ \\
\hline \multicolumn{2}{|l|}{ Drug use } \\
\hline Yes & $6(15.8)$ \\
\hline No & $32(84.2)$ \\
\hline \multicolumn{2}{|c|}{ Genital infection symptom } \\
\hline Pruritus & $2(5.3)$ \\
\hline Irritation & $1(2.6)$ \\
\hline Genital secretion & $7(18.4)$ \\
\hline Pelvic pain & $1(2.6)$ \\
\hline Asymptomatic & $5(13.2)$ \\
\hline Some of these signs & $22(57.9)$ \\
\hline \multicolumn{2}{|l|}{ Husband education } \\
\hline Cycles & $14(36.8)$ \\
\hline Diploma & 11 (29) \\
\hline College & $13(34.2)$ \\
\hline \multicolumn{2}{|l|}{ Contraception } \\
\hline Drug & $3(7.9)$ \\
\hline Condom & $14(36.8)$ \\
\hline Natural & $13(34.2)$ \\
\hline Tubectomy & $2(5.3)$ \\
\hline IUD & $1(2.6)$ \\
\hline Ampulla & $1(2.6)$ \\
\hline \multicolumn{2}{|l|}{ Marriage age } \\
\hline $1-10 y$ & 22 (57.9) \\
\hline $11-20 y$ & $11(28.9)$ \\
\hline $21-30 y$ & $5(13.2)$ \\
\hline \multicolumn{2}{|l|}{ Child number } \\
\hline $0-2$ & 31 (81.6) \\
\hline $3-5$ & 7 (18.4) \\
\hline \multicolumn{2}{|l|}{ Antibiotic usage } \\
\hline Yes & $9(23.7)$ \\
\hline No & $29(76.3)$ \\
\hline \multicolumn{2}{|l|}{ Hospitalization } \\
\hline Yes & $7(18.4)$ \\
\hline No & 31 (81.6) \\
\hline Medical device & \\
\hline Yes & $2(5.3)$ \\
\hline No & $36(94.7)$ \\
\hline Preterm delivery & \\
\hline Yes & $1(2.6)$ \\
\hline No & $37(97.4)$ \\
\hline
\end{tabular}


Table 2. The Ureaplasma urealyticum Antimicrobial Susceptibility Profile ( $\mathrm{n}=38$ )

\begin{tabular}{|c|c|c|c|c|c|c|c|c|c|}
\hline & DOT & TET & OFL & CIP & JOS & ERY & CLA & AZT & PRI \\
\hline Susceptible & $35(92.1)$ & $35(92.1)$ & $25(65.8)$ & $17(44.7)$ & $22(57.9)$ & $23(60.5)$ & $27(71.1)$ & $25(65.8)$ & $23(60.5$ \\
\hline Intermediate & $0(0)$ & $1(2.6)$ & 11 (28.9) & $14(36.8)$ & $11(28.9)$ & 7 (18.4) & $4(10.5)$ & $6(15.8)$ & - \\
\hline Resistant & $3(7.9)$ & $2(5.3)$ & $2(5.3)$ & 7 (18.4) & $5(13.2)$ & $8(21.1)$ & $7(18.4)$ & 7 (18.4) & $15(39.5$ \\
\hline Total & $38(100)$ & $38(100)$ & $38(100)$ & $38(100)$ & $38(100)$ & $38(100)$ & $38(100)$ & $38(100)$ & $38(100)$ \\
\hline
\end{tabular}

Note. DOT: Doxycycline; TET: Tetracycline; OFL: Ofloxacin; CIP: Ciprofloxacin; JOS: Josamycin; ERY: Erythromycin; CLA: Clarithromycin; AZT: Azithromycin; PRI: Pristinamycin.

Table 3. Antimicrobial Resistance Pattern of Ureaplasma urealyticum Isolates With $\geq 10^{4} \mathrm{CFU}$ and $<10^{4} \mathrm{CFU}$

\begin{tabular}{|c|c|c|c|c|c|c|c|c|c|}
\hline & ERY & CLA & AZT & CIP & OFL & DOT & TET & JOS & PRI \\
\hline$\geq 104$ CFU/specimen $(n=33)$ & $45.4 \%$ & $33.3 \%$ & $39.4 \%$ & $63.6 \%$ & $39.4 \%$ & $9.1 \%$ & $9.1 \%$ & $48.5 \%$ & $45.4 \%$ \\
\hline$<104$ CFU/specimen $(n=5)$ & $100 \%$ & $100 \%$ & $100 \%$ & $100 \%$ & $100 \%$ & $100 \%$ & $100 \%$ & $100 \%$ & $100 \%$ \\
\hline
\end{tabular}

Note. ERY: Erythromycin; CLA: Clarithromycin; AZT: Azithromycin; OFL; Ofloxacin; CIP: Ciprofloxacin; DOT: Doxycycline; TET: Tetracycline; JOS: Josamycin; PRI: Pristinamycin.

birth, postpartum infections, urogenital diseases, and adverse pregnancy outcomes (11). Given the frequent implementation of diagnostic techniques that only recognize causative organisms, these bacterial infections are generally treated by TETs, macrolides (i.e., AZI, ERY, and clarithromycin), or fluoroquinolonesin empirical therapy (12). Nevertheless, information regarding the antimicrobial sensitivity profile of genital mycoplasmas is limited, and regional statistics are exclusively required to establish efficient treatments.

Considering antibiotic susceptibility outcomes, the results of the present research showed that $U$. urealyticum isolates exhibited a high rate of resistance to fluoroquinolones (55.2\% of the isolates were resistant or intermediate-susceptible to CIP), which is consistent with the results of another research. Overall, the highest antibiotic resistance rates have been reported against fluoroquinolones in most geographical regions $(12,13)$. Two Chinese analyses reported fluoroquinolone resistance rates of $40 \%$ for Ureaplasma spp. (12). The resistance is mostly because of antibiotics overuse in different industries and human communities (e.g., for different infections including urinary and respiratory systems diseases), which contributes to the selection of drug-resistant $U$. urealyticum $(14,15)$. Given that many CIP-resistant isolates are susceptible to TETs (i.e., TET and DOT), treatment should involve monotherapy or combination therapy including TETs.

In this investigation, $42.1 \%$ of the surveyed strains were non-susceptible to JOS, but the prevalence of resistance to PRI was lower (39.5\%). Further, the rate of JOS resistance was much higher compared to a study on the rate of JOS resistant $U$. urealyticum (58.7\%) in Bern, Switzerland (12). The same increase in the rate of JOS resistance $U$. urealyticum has been reported earlier in different studies reported from several regions of the world (16). In the present study, $39.4 \%$ of 33 U. urealyticum isolates with $\geq 10^{4} \mathrm{CFU}$ were resistant to OFL while all isolates with
$<10^{4}$ CFU were susceptible to all tested antibiotics.

As revealed, $9.1 \%$ of the strains were insensitive to DOT and TET. These results are in line with prior studies from Switzerland and China which reported that all strains were sensitive to these antibiotics $(12,17)$. Therefore, TET resistant strains emerging in our investigation are really alarming. The important risk for drug resistance emerging in $U$. urealyticum has potential clinical sequels in therapeutic guidelines. In other fields such as plants, poultries, food animals, fish, and other sources, antimicrobial agents are applied for various purposes potentially leading to emerging insensitive isolates.

Furthermore, a high rate of ERY-resistant $U$. urealyticum (i.e., 39.5\%) was observed in contrast with the rates from Romania, which was reported $16.09 \%$. Resistance to AZI and CLA has been reported at 8.05\%, and 9.19\%, respectively in Romania as well (18).

Pristinamycin resistant isolates (39.5\%) detected in our study are quite alarming. Our data contradict those of other studies from different geographical regions, presenting the high sensitivity to this drug among $U$. urealyticum isolates $(12,19,20)$.

In Chinese studies, the prevalence rates of TET and macrolide non-susceptible strains were $10 \%$ and 30\%, respectively $(21,22)$. In Croatia, $U$. urealyticum strains showed resistance rates of $3 \%, 8 \%$, and $22 \%$ for DOT, AZI, and OFL, respectively (13). In South Africa, the non-susceptibility rates of ERY, moxifloxacin, TET, and levofloxacin were $80 \%, 2 \%, 73 \%$, and $41 \%$, respectively (23). These contradictions in resistance rates among the aforementioned reports could be due to the usage of different techniques and criteria for the interpretation of susceptibility results.

Although the emerging resistance to TETs among our isolates is alarming, the reports by the Schneider et al indicated that the therapeutic protocols for urogenital infections caused by $U$. urealyticum include DOT, TET, and clarithromycin (12), leading to efficient outcomes in 
most respective patients.

Research on other pathogenic bacteria such as foodborne pathogens in women is necessary. Hormonal changes that occur in pregnancy reduce cell-dependent immunity, thus raising the susceptibility of pregnant women to some microbial infections. Foodborne diseases may be worrying in pregnancy and can lead to preterm delivery or abortion and serious sequelae in newborn babies (24-29).

\section{Conclusions}

Culture is still the most widely used means for the isolation and identification of genital Ureaplasma spp. in human samples, and it remains the accepted reference standard. These bacteria are highly sensitive to unfavorable conditions (i.e., heat and drying) in the environment, thus great attention must be paid to ensure proper specimen collection and transport. Dacron, polyester, or calcium alginate swabs with plastic shafts or aluminum are more suitable. To collect swabs, we should select the sites where most cells can be obtained because Ureaplasma spp. are cell-associated.

\section{Authors' Contribution}

All authors contributed to this study equally.

\section{Conflict of Interests}

Authors declare that they have no conflict of interests.

\section{Financial Support}

This study was supported by Gonabad University of Medical Sciences, Gonabad, Iran.

\section{Acknowledgments}

The authors would like to thank the Clinical Research Development Unit, Allame Bohlool Hospital, Gonabad University of Medical Sciences (Gonabad, Iran) for the cooperation in sampling. Our special thanks go to $\mathrm{H}$. Khajehei for language editing of the manuscript.

\section{References}

1. Razin S, Yogev D, Naot Y. Molecular biology and pathogenicity of mycoplasmas. Microbiol Mol Biol Rev. 1998;62(4):10941156.

2. Waites KB, Xiao L, Paralanov V, Viscardi RM, Glass Jl. Molecular methods for the detection of Mycoplasma and Ureaplasma infections in humans: a paper from the 2011 William Beaumont Hospital Symposium on molecular pathology. J Mol Diagn. 2012;14(5):437-450. doi:10.1016/j. jmoldx.2012.06.001

3. Paralanov V, Lu J, Duffy LB, et al. Comparative genome analysis of 19 Ureaplasma urealyticum and Ureaplasma parvum strains. BMC Microbiol. 2012;12:88. doi:10.1186/14712180-12-88

4. Baseman JB, Tully JG. Mycoplasmas: sophisticated, reemerging, and burdened by their notoriety. Emerg Infect Dis. 1997;3(1):21-32. doi:10.3201/eid0301.970103

5. Viscardi RM, Kallapur SG. Role of Ureaplasma respiratory tract colonization in bronchopulmonary dysplasia pathogenesis: current concepts and update. Clin Perinatol. 2015;42(4):719738. doi:10.1016/j.clp.2015.08.003

6. Bloomfield P. Update on emerging infections: news from the
Centers for Disease Control and Prevention. Update to CDC's Sexually Transmitted Diseases Treatment Guidelines, 2006: fluoroquinolones no longer recommended for treatment of gonococcal infections. Ann Emerg Med. 2007;50(3):232-235. doi:10.1016/j.annemergmed.2007.06.013

7. Kataoka S, Yamada T, Chou K, et al. Association between preterm birth and vaginal colonization by mycoplasmas in early pregnancy. J Clin Microbiol. 2006;44(1):51-55. doi:10.1128/jcm.44.1.51-55.2006

8. Beeton ML, Chalker VJ, Jones LC, Maxwell NC, Spiller OB. Antibiotic resistance among clinical Ureaplasma isolates recovered from neonates in England and Wales between 2007 and 2013. Antimicrob Agents Chemother. 2016;60(1):52-56. doi:10.1128/aac.00889-15

9. García-Castillo M, Morosini Ml, Gálvez M, Baquero F, del Campo R, Meseguer MA. Differences in biofilm development and antibiotic susceptibility among clinical Ureaplasma urealyticum and Ureaplasma parvum isolates. J Antimicrob Chemother. 2008;62(5):1027-1030. doi:10.1093/jac/dkn337

10. Jin H, Qi C, Zou Y, et al. Biochanin A partially restores the activity of ofloxacin and ciprofloxacin against topoisomerase IV mutation-associated fluoroquinolone-resistant Ureaplasma species. J Med Microbiol. 2017;66(11):1545-1553. doi:10.1099/jmm.0.000598

11. Larsen B, Hwang J. Mycoplasma, Ureaplasma, and adverse pregnancy outcomes: a fresh look. Infect Dis Obstet Gynecol. 2010;2010. doi:10.1155/2010/521921

12. Schneider SC, Tinguely R, Droz S, et al. Antibiotic susceptibility and sequence type distribution of Ureaplasma species isolated from genital samples in Switzerland. Antimicrob Agents Chemother. 2015;59(10):6026-6031. doi:10.1128/ aac.00895-15

13. Vargović $M$, Pasini $M$, Papić $N$, et al. Antimicrobial susceptibility of Ureaplasma urealyticum and Mycoplasma hominis. Sex Transm Infect. 2014;90(1):69. doi:10.1136/ sextrans-2013-051413

14. Kamiya $Y$, Shimada $Y$, Ito $S$, et al. Analysis of the quinoloneresistance determining region of the gyrA gene and the analogous region of the parC gene in Ureaplasma parvum and Ureaplasma urealyticum detected in first-void urine of men with non-gonococcal urethritis. J Antimicrob Chemother. 2013;68(2):480-482. doi:10.1093/jac/dks417

15. Kawai $Y$, Nakura $Y$, Wakimoto $T$, et al. In vitro activity of five quinolones and analysis of the quinolone resistancedetermining regions of gyrA, gyrB, parC, and parE in Ureaplasma parvum and Ureaplasma urealyticum clinical isolates from perinatal patients in Japan. Antimicrob Agents Chemother. 2015;59(4):2358-2364. doi:10.1128/aac.0426214

16. Xie X, Zhang J. Trends in the rates of resistance of Ureaplasma urealyticum to antibiotics and identification of the mutation site in the quinolone resistance-determining region in Chinese patients. FEMS Microbiol Lett. 2006;259(2):181-186. doi:10.1111/j.1574-6968.2006.00239.x

17. Wang QY, Li RH, Zheng LQ, Shang XH. Prevalence and antimicrobial susceptibility of Ureaplasma urealyticum and Mycoplasma hominis in female outpatients, 20092013. J Microbiol Immunol Infect. 2016;49(3):359-362. doi:10.1016/j.jmii.2014.06.007

18. Mihai M, Valentin N, Bogdan D, Carmen CM, Coralia B, Demetra S. Antibiotic susceptibility profiles of Mycoplasma hominis and Ureaplasma urealyticum isolated during a population-based study concerning women infertility in northeast romania. Braz J Microbiol. 2011;42(1):256-260. doi:10.1590/s1517-83822011000100032

19. Lee MY, Kim MH, Lee WI, Kang SY, Jeon YL. Prevalence 
and antibiotic susceptibility of Mycoplasma hominis and Ureaplasma urealyticum in pregnant women. Yonsei Med J. 2016;57(5):1271-1275. doi:10.3349/ymj.2016.57.5.1271

20. Bayraktar MR, Ozerol IH, Gucluer N, Celik O. Prevalence and antibiotic susceptibility of Mycoplasma hominis and Ureaplasma urealyticum in pregnant women. Int J Infect Dis. 2010;14(2):e90-95. doi:10.1016/j.ijid.2009.03.020

21. Song T, Ye A, Xie X, et al. Epidemiological investigation and antimicrobial susceptibility analysis of Ureaplasma species and Mycoplasma hominis in outpatients with genital manifestations. J Clin Pathol. 2014;67(9):817-820. doi:10.1136/jclinpath-2014-202248

22. Wang QY, Li RH, Zheng LQ, Shang XH. Prevalence and antimicrobial susceptibility of Ureaplasma urealyticum and Mycoplasma hominis in female outpatients, 20092013. J Microbiol Immunol Infect. 2016;49(3):359-362. doi:10.1016/j.jmii.2014.06.007

23. Redelinghuys MJ, Ehlers MM, Dreyer AW, Lombaard HA, Kock MM. Antimicrobial susceptibility patterns of Ureaplasma species and Mycoplasma hominis in pregnant women. BMC Infect Dis. 2014;14:171. doi:10.1186/1471-2334-14-171

24. Mardaneh J, Soltan-Dallal MM. Isolation and identification of $E$. cowanii from powdered infant formula in NICU and determination of antimicrobial susceptibility of isolates. Iran J Pediatr. 2014;24(3):261-266.

25. Anvarinejad M, Pouladfar GR, Pourabbas B, et al. Detection of Salmonella spp. with the BACTEC 9240 Automated Blood
Culture System in 2008 - 2014 in Southern Iran (Shiraz): Biogrouping, MIC, and Antimicrobial Susceptibility Profiles of Isolates. Jundishapur J Microbiol. 2016;9(4):e26505. doi:10.5812/jjm.26505

26. Mardaneh J, Soltan Dallal MM, Taheripoor M, Rajabi Z. Isolation, identification and antimicrobial susceptibility pattern of Tatumella ptyseos strains isolated from powdered infant formula milk consumed in neonatal intensive care unit: first report from Iran. Jundishapur J Microbiol. 2014;7(6):e10608. doi:10.5812/jjm.10608

27. Pourabbas B, Ziyaeyan M, Alborzi A, Mardaneh J. Efficacy of measles and rubella vaccination one year after the nationwide campaign in Shiraz, Iran. Int J Infect Dis. 2008;12(1):43-46. doi:10.1016/j.ijid.2007.03.013

28. Hassanzadeh P, Mardaneh J, Motamedifar M. Conventional agar-based culture method, and Nucleic Acid Amplification Test (NAAT) of the сррB gene for detection of Neisseria gonorrhea in pregnant women endocervical swab specimens. Iran Red Crescent Med J. 2013;15(3):207-211. doi:10.5812/ ircmj.3726

29. Pourabbas B, Rezaei Z, Mardaneh J, Shahian M, Alborzi A. Prevalence of Chlamydia trachomatis and Neisseria gonorrhoeae infections among pregnant women and eye colonization of their neonates at birth time, Shiraz, Southern Iran. BMC Infect Dis. 2018;18(1):477. doi:10.1186/s12879018-3382-4

(C) 2021 The Author(s); This is an open-access article distributed under the terms of the Creative Commons Attribution License (http:// creativecommons.org/licenses/by/4.0), which permits unrestricted use, distribution, and reproduction in any medium, provided the original work is properly cited. 